\title{
A NOVEL MEMS TUNABLE CAPACITOR BASED ON ANGULAR VERTICAL COMB DRIVE ACTUATORS
}

\author{
Hung Nguyen, Dooyoung Hah, Pamela R. Patterson, Wibool Piywattanametha, Ming C. Wu \\ Department of Electrical Engineering \\ University of California, Los Angeles, CA 90095, U.S.A.
}

\author{
Rumin Chao \\ National Cheng Kung University \\ TAIWAN
}

\begin{abstract}
We report on the design, fabrication, and measurement of a novel MEMS (MicroElectroMechanical System) tunable capacitor with angular vertical comb-drive (AVC) actuators. The AVC allows continuous rotation and therefore large tuning ratio without pull-in. The device fabrication process is relatively simple, consisting of a single deep reactive ion etching step followed by release and out-of-plane assembly of the angular combs. We also report a new 3-D self-assembly process using reflowed Dow Chemical's BCB (Cyclotene) hinges, which are more robust than conventional photoresist hinges. The use of glass substrate has greatly reduced parasitic capacitances. A tuning ratio of 4.2 has been achieved with $10-\mu \mathrm{m}$-thick device layer. Our theoretical simulation shows that larger tuning ratio can be achieved with thicker device layers and further optimization of the design.
\end{abstract}

\section{INTRODUCTION}

MEMS (MicroElectroMechanical System) tunable capacitors have recently garnered significant interest due to their inherent performance advantages over conventional solid-state varactors; They generally exhibit larger tuning range and higher quality factor, Q, by reason of lower resistive and insertion losses.

Most MEMS tunable capacitors employ gap-closing electrostatic actuation, which offers relatively short response times and requires low power with little or no heat generation [1]. However, gap-closing actuators also suffer from some fundamental limitations. These actuators exhibit a pull-in phenomenon at one third of the gap distance between conductors, which limits the tuning ratio to $1.5(50 \%)$.

There have been several attempts to overcome this theoretical limit via modifications to the gap-closing design [2]. Nevertheless, the tuning ratios achieved are still very modest. The use of comb drive actuators has made it possible to achieve a very large tuning ratio [3]. While lateral comb drives do not suffer from pull-in, the capacitance tuning relies on the lateral motion of the movable fingers. Hence, the tuning ratio is limited by the maximum separation of these fingers and their overall lengths. In this paper, we propose a novel MEMS tunable capacitor based on angular vertical comb-drive (AVC) actuators [4]. Unlike conventional comb-drives that have limited lateral displacements and require larger area for higher tuning, AVCs have the potential for larger tuning range through rotation. This reduces the dependence on the device size. A tuning ratio of 4.2 has been successfully achieved. We have also

\footnotetext{
Travel support has been generously provided by the

Transducers Research Foundation and by the DARPA

MEMS and DARPA BioFlips programs
}

performed detailed theoretical simulation of the AVC tunable capacitor. Our theory shows that larger tuning ratio can be achieved with optimized device structures.

\section{DESIGN PRINCIPLE}

Figure 1 shows the schematics of the AVC tunable capacitor at zero bias (maximum capacitance) and actuated (minimum capacitance) configurations. The MEMS tunable capacitor utilizes two sets of interdigitated comb fingers to vary the capacitance. The shorter suspended DC comb fingers are set at an initial angle prescribed by the design. With a voltage bias between the suspended and fixed fingers, the electric field pulls the suspended fingers towards the fixed fingers, which in turn, rotates the longer RF fingers that are located at the opposite end of the device. The change in the overlapping area of the RF comb fingers due to the rotation tunes the total capacitance.
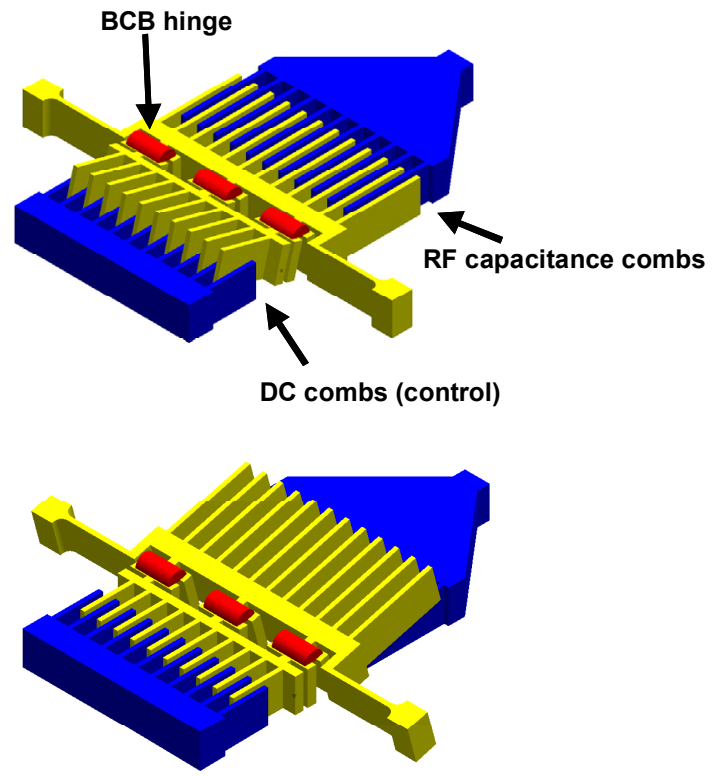

Figure 1. Schematic of tunable capacitor at maximum capacitance (top) and minimum capacitance (bottom).

For small initial tilt angles, the AVC actuator exhibits continuous rotation just like conventional lateral comb drives in lateral translation. When the initial tilt angle exceeds a critical angle, electrostatic pull-in between the tilted comb and the fixed comb starts to appear. This critical angle depends on the geometry of the device. Careful consideration of the device 
dimensions are taken to avoid the pull-in phenomenon. Equation (1) describes the condition for which pull-in can occur.

$$
P I(\theta)=\frac{\partial C}{\partial \theta}-\theta \frac{\partial^{2} C}{\partial \theta^{2}}
$$

where $\mathrm{C}$ is the capacitance of the actuator combs and $\theta$ is the angle of rotation. Equation (1) is derived from the equilibrium equation between the electrostatic and restoring mechanical torques. To avoid pull-in, Eq. (1) must be positive for all angles. The equation can be rewritten in terms of comb finger dimensions to determine the maximum finger lengths for actuator combs, as shown in Eq. (2):

$$
l_{\text {max }}=\frac{3}{2} \frac{t_{\text {finger }}}{\theta_{\text {initial }}}-l_{\text {offset }}
$$

where $l_{\max }$ is the maximum allowed overlapped finger length of DC comb without pull-in and $l_{\text {offset }}$ is the distance from the axis of rotation to base of the DC moving fingers. The device thickness, which in this case is $10 \mu \mathrm{m}$, is represented by $t_{\text {finger }}$. From Eq. (2) and the predetermined reflow angle $\theta_{\text {initial }}$, optimal dimensions for the tunable capacitor for maximum tuning range can be found. It should be pointed out that the RF comb fingers can be much longer than the DC fingers, unlike in lateral combdrive devices where RF fingers needs to be shorter. This is utilized to increase the capacitance and to optimize the tuning ratio. The only limit on the length of RF fingers is lateral bending and stiction of the fingertips.

\section{FABRICATION}

Figure 2 shows the details of the fabrication process flow. The tunable capacitor is fabricated on glass substrate to reduce parasitic capacitance. We have chosen borofloat glass (BSG) substrate because its faster etch rate and . This greatly facilitates the releasing step. The silicon device layer from an silicon-oninsulator (SOI) wafer is bonded face down to the BSG substrate by anodic bonding. Prior to bonding, the SOI and the glass wafers are thoroughly cleaned and dehydrated to ensure intimate contact between the wafers. The wafers are then anodically bonded at $400^{\circ} \mathrm{C}$ and $700 \mathrm{~V}$ (Fig. 2(a)). Bonding times vary depending on applied voltage. Lower voltages require longer bonding time but produces a more effective bonding and fewer voids. The thermal expansion coefficient (TEC) of BSG is also well matched to that of $\mathrm{Si}$ below $400^{\circ} \mathrm{C}$, which greatly reduces the stress on the silicon device layer. The SOI substrate is subsequently removed by a combination of mechanical and chemical etches (Fig. 2(b)). The exposed buried oxide is also etched in a diluted solution of buffered hydrofluoric acid (BOE). Once the SOI substrate and buried oxide layer are removed, the $10-\mu \mathrm{m}$-thick silicon film is patterned and deep reactive ion etched (DRIE) to form the device structure (Fig. 2(c)). Next, photosensitive BCB (Cycotene) hinges are patterned to physically connect the suspended RF and DC fingers (Fig. 2(d)). Without curing the $\mathrm{BCB}$ hinges, the glass substrate is then etched in $49 \%$ hydrofluoric acid to release the suspended fingers (Fig. 2(e)). Release time is approximately six minutes. The hinges are then cured at 400 degrees in a nitrogen overpressured oven for twenty minutes. The movable DC comb fingers are assembled to an initial angle above the substrate plane (Fig. 2(f)). At the same time the high temperatures removes excess solvent from the release. The final process step involves the metallization over the entire sample with $2500 \AA$ of sputtered aluminum for better conductivity. Sputtered aluminum provides better step coverage particularly at the sidewalls of the $10-\mu \mathrm{m}-$ thick comb fingers.

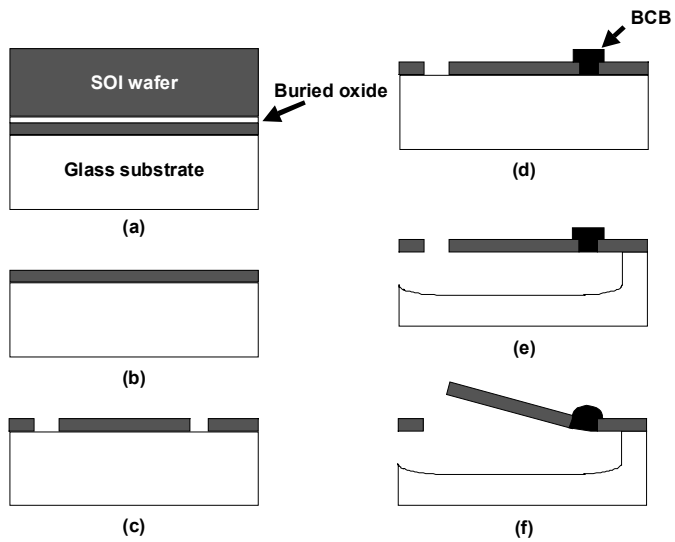

Figure 2. Fabrication process flow.

The combination of high temperature and HF exposure causes the BCB hinges to reflow. The reflow process allows automatic assembly of the DC fingers to an initial reflow angle. Limiters included on the hinge design limits the rotation to approximately 10 degrees. A similar process had been done with conventional photoresist [5]. However, BCB is far more attractive due to its chemical and thermal stability [6]. Unlike conventional photoresist, uncured $\mathrm{BCB}$ can withstand prolonged exposure to $\mathrm{HF}$ and solvents without evidence of delaminating or degradation. A scanning electron micrograph (SEM) of the device including the suspended fingers upon release and assembly is shown in Fig. 3.

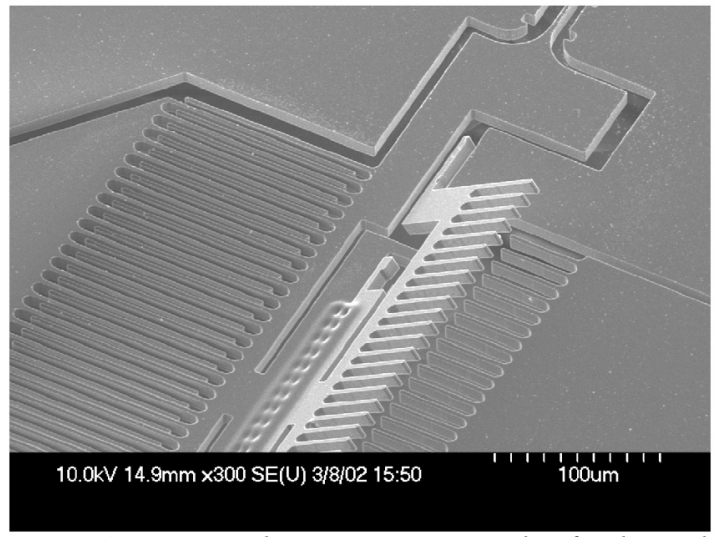

Figure 3. Scanning electron micrograph of released and assembled comb fingers.

\section{EXPERIMENTAL RESULTS}

Our initial design for the MEMS tunable capacitor exhibited a tuning ratio of 1.67. While this value exceeded the theoretical limit of 1.5 for parallel plate capacitors, the performance was far from satisfactory. After detailed examination, we have concluded that the smaller than expected tuning ratio is due to the sagging of the suspended comb fingers along the length of the device. The sagging results in finite overlap of the RF comb fingers at maximum rotation. 
In our second-generation devices, we shorten the length and increase the width of the torsion spring. Since the bending spring constant is inversely proportional to the cube of the length, this greatly reduces the sagging with slight increase of actuation voltage. Figure 4 shows the interferometric image of the modified device at maximum bias. The DC movable fingers are now aligned with the fixed fingers without any evidence of sagging. The residue overlapping capacitance between RF fingers is greatly reduced. This leads to a $262 \%$ increase in the tuning ratio to 4.2 .

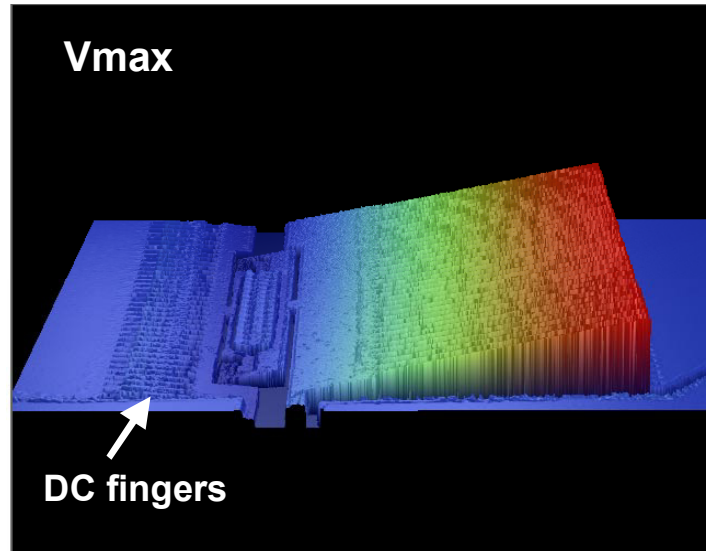

Figure 4. Interferometric image of improved device at maximum voltage. The $R F$ fingers are rotated upward.

The capacitance and the $\mathrm{S}$ parameters are measured with a Hewlett Packard network analyzer $8510 \mathrm{C}$ and a high frequency coplanar waveguide probe with ground-signal-ground configuration. The measurements are calibrated for two-port measurements. Maximum capacitance at zero bias is measured at $1.34 \mathrm{pF}$. At maximum voltage, the capacitance is reduced to $0.32 \mathrm{pF}$. Figure 5 shows the capacitance-versus-voltage (CV) measurement of the tunable capacitor. As expected, the device does not show signs of pull-in. The capacitance reduces continuously as the voltage increases to $60 \mathrm{~V}$. Beyond $60 \mathrm{~V}$, the capacitance saturates and decreases very slowly. The S11 parameters are shown in Fig. 6 at the minimum and the maximum capacitance, respectively. The quality factor $\mathrm{Q}$ is measured by extrapolating the impedance values with respect to frequency from the network analyzer. The quality factor, Q, is measured to be 71 and 8.7 at $1 \mathrm{GHz}$ for minimum and maximum capacitances, respectively.

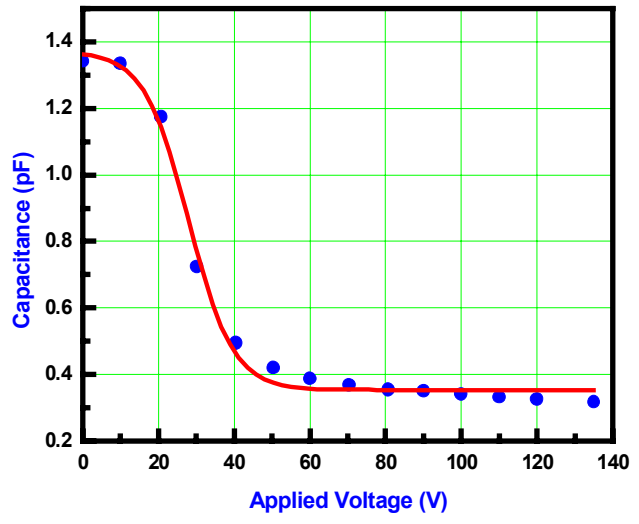

Figure 5. C-V measurement of the tunable capacitor.

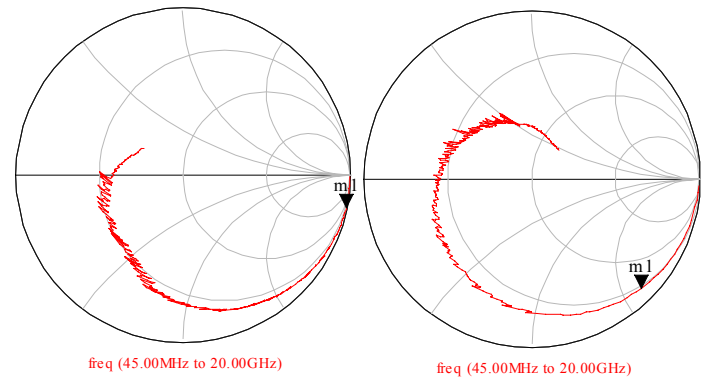

Figure 6. Smith chart showing S11 at (left) minimum and (right) maximum capacitance

The transfer curve that corresponds to the $\mathrm{C}-\mathrm{V}$ measurement is shown in Fig. 7. It shows the angles of both the DC and RF comb fingers. The suspended DC fingers have an initial reflow angle of 8 degrees. Maximum rotation of 3 degrees occurs at 60 V. In principle, full rotation $\left(8^{\circ}\right)$ should be reached at sufficiently high voltage for AVC actuators. A close examination of the device operation under microscope reveals that the rotating structure hits an intruding beam at $3^{\circ}$, thus preventing the fingers from further rotation. By modifying the design, we believe larger rotation angle can be achieved.

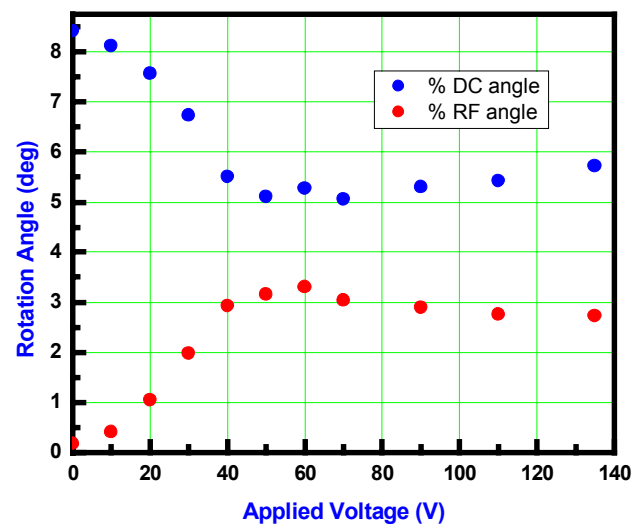

Figure 7. Transfer curve of $R F$ and DC fingers with varying bias voltage.

We have performed theoretical modeling of the AVC tunable capacitor to investigate various parameter ranges for optimizing its performance. We found that the most effective way to increase the tuning ratio and reducing voltage is to increase the device layer thickness. Similar trend is also observed in tunable capacitors with lateral comb drive actuators. Borwick, et. al have shown that increasing the device thickness from $30 \mu \mathrm{m}$ to $80 \mu \mathrm{m}$ raises the tuning range to from 3 to 8 [7]. Migration towards thicker silicon film allows for larger tuning ratios as maximum capacitance between the comb fingers is increased compared to the fringe capacitance when the comb fingers are separated. Figure 8 shows the maximum tuning ratio for a $25-\mu \mathrm{m}$-thick device with 10 degree initial tilt angle. It is possible to obtain a tuning ratio of at least 15 for a given finger length. As with the tuning ratio, the quality factor can be improved with the use of highly resistive SOI wafers to reduce resistive loss from the silicon device layers. 


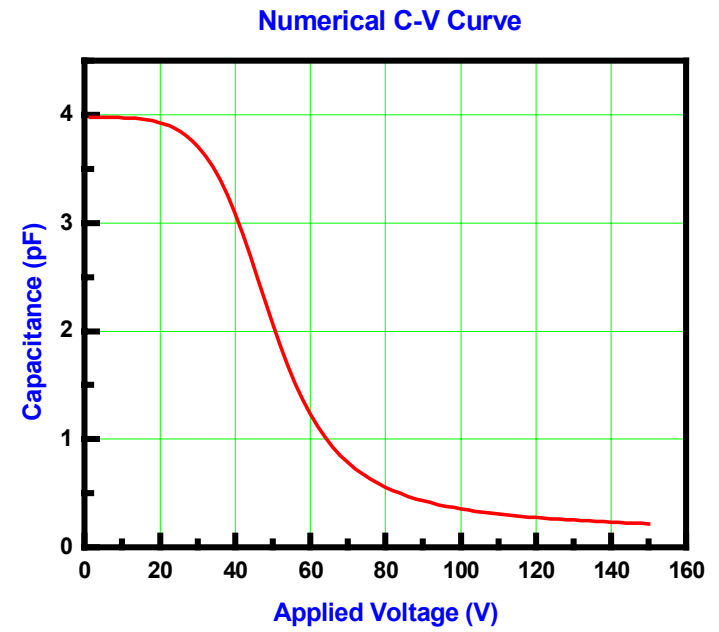

Figure 8. Theoretic C-V curve for 25- $\mu$ m-thick tunable capacitor

\section{CONCLUSION}

We have proposed and successfully fabricated a novel MEMS tunable capacitor with angular vertical comb-drive (AVC) actuators. Continuous tuning without pull-in has been successfully achieved. A tuning ratio of 4.2 has been measured at $1 \mathrm{GHz}$ for a $10-\mu \mathrm{m}$-thick device. This is larger than that of lateral comb drive devices with similar device layer thickness. Larger tuning ratio and high $\mathrm{Q}$ value can be achieved with thicker device layers.

\section{AKNOWLEDGMENT}

The authors would like to thank Drs. Robert Y. Loo, Jim Shaffner, Dan Sivenpiper, and Greg Tangonan of HRL for their valuable discussions. Special thanks also goes to Sagi Mathai and Sanjeev Murthy of UCLA for their assistance in microwave measurements.

This project is supported in part by HRL Laboratories under the University of California MICRO program, and the DARPA Contract N66001-99-C-8635. Travel support has been provided by the Transducers Research Foundation and by the DARPA MEMS and DARPA BioFlips programs.

\section{REFERENCES}

1. Dec, A.; Suyama, K., "A 1.9-GHz CMOS VCO with micromachined, vol.35, (no.8), IEEE, Aug. 2000. p.1231-7.

2. Jun Zou; Chang Liu; Schutt-Aine, J.; Jinghong Chen; SungMo Kang, "Development of a wide tuning range MEMS tunable capacitor for wireless communication systems." International Electron Devices Meeting 2000

3. Yao, J.J.; Park, S.; Anderson, R.; DeNatale, J., "A low power/low voltage electrostatic actuator for RF MEMS applications." Solid-State Sensor and Actuator Workshop, Hilton Head Island, SC, USA, 4-8 June 2000.

4. P. Patterson, D. Hah, H. Chang, H. Toshiyoshi, M.C. Wu, "An Angular Vertical Comb Drive Actuator for Scanning Micromirrors", 2001 International Conference on Optical MEMS, Okinawa, Japan,p.25.
5. Syms, R.R.A.; Gormley, C. V; Blackstone, S., "Improving yield, accuracy and complexity in surface tension self-assembled MOEMS," Sensors and Actuators A (Physical), vol.A88, (no.3), Elsevier, 5 March 2001. p.273-83.

6. Tuantranont, A.; Bright, V.M.; Zhang, J.; Zhang, W.; Neff, J.A.; Lee, Y.C. "Optical beam steering using MEMScontrollable microlens array" Solid-State Sensor and Actuator Workshop, Hilton Head Island, SC, USA, 4-8 June 2000.

7. R.Borwick, P. Stupar, J. DeNatale, R. Anderson, C. Tsai, and $\mathrm{K}$. Garrett, "A High Q, Large Tuning Range, Tunable Capacitor for RF Applications," MEMS 2002, Las Vegas, NV, USA 